\title{
Thrombolysis in Cerebral Infarction Scoring at the Core Lab
}

\author{
Mohammed A. Almekhlafi*, Mayank Goyal ${ }^{\star}$, Henk A. Marquering ${ }^{\dagger} \ddagger$, Charles B. Majoie ${ }^{\dagger}$, David S. Liebeskind ${ }^{\S}$ \\ Department of Radiology, University of Calgary*, Calgary, Canada, Department of Radiology and Nuclear Medicine, Academic Medical Center ${ }^{\dagger}$, \\ Amsterdam; Biomedical Engineering and Physics, Academic Medical Center ${ }^{\ddagger}$, Amsterdam, the Netherlands, Neurovascular Imaging Research \\ Core and UCLA Stroke Center ${ }^{\S}$, Los Angeles, CA, USA
}

The success of endovascular thrombectomy (EVT) procedure centers on achieving fast and complete reperfusion. Angiographic assessment of reperfusion was originally reported using the Thrombolysis in Cerebral Infarction (TICI) scoring published in 2003. Since then, the TICI scoring underwent multiple modifications leading to different interpretations which negatively impacted its reliability. We discuss the implications and nuances of unifying angiographic reporting using the expanded TICI (eTICI) scoring system which was used to report the angiographic images of patients enrolled in the Highly Effective Reperfusion Evaluated in Multiple Endovascular Stroke Trials collaboration. We propose the use of this eTICI scoring as a reference in core labs of future EVT trials and in clinical practice. We also discuss solutions to help to bridge the gap that currently exists due to the different interpretations of the TICI score. Unified reporting of the angiographic outcome of EVT procedures is paramount to advancing the field and improving the understanding of predictors of functional outcome especially with the current high rates of successful reperfusion.

J Neurosonol Neuroimag 201\$;10(2):95-99

Key Words: Strokes; Thrombectomies; Reperfusion; Ischemia
Received: November 1, 2018

Revised: November 23, 2018

Accepted: November 27, 2018

Address for correspondence: Mohammed A. Almekhlafi

Department of Radiology, University of Calgary, 140329 St. NW, Calgary, AB T2N 2T9, Canada

Tel: +1-403-944-1883

Fax: +1-403-944-2611

E-mail: mohammed.almekhlafi@ucalgary.ca
Core lab adjudication of angiographic reperfusion using the Thrombolysis in Cerebral Infarction (TICI) scale has been an integral component of endovascular stroke therapy trials. ${ }^{1-7}$ Despite the specific definitions published on the TICI scoring and its fundamental role as a quality measure in certifying stroke centers, ${ }^{6}$ its application at local stroke centers continues to vary. ${ }^{8}$ This variability prompted multiple revisions and modifications of the scale. At the same time, the implied equivalence of success in endovascular therapy with more complete reperfusion (i.e., higher TICI scores) prompted some trials to reduce the scale to a dichotomous outcome. For instance, the use of a threshold score of $2 \mathrm{~B}$ or more in the modified TICI (mTICI) $\left(\geq_{50} \%\right.$ reperfusion of the territory downstream of the occlusion) has been widely used to define successful reperfusion after endovascular therapy (Table 1). ${ }^{9}$ This enforced an inherent bias to overcall TICI scores and subsequently inflated the number of successful procedures. It is not uncommon to see a TICI score of 3 ascribed despite subtle filling defects or delay in contrast opacification on final angiography images. A final TICI score of 3 in these cases ignores the potential interaction between some of these findings with individual patient pathophysiology and biological factors such as collateral status that may alter the degree and topography of reperfusion. ${ }^{10}$ Furthermore, the tendency for overcalling TICI scores widened the gap between the neurological outcomes of patients and the described angiographic success. A recent modification incorporating TICI $2 \mathrm{c}$ (also known as the expanded TICI [eTICI]; Fig. 1) have been adopted by the core lab in the Highly Effective Reperfusion Evaluated in Multiple Endovascular Stroke Trials (HERMES) collaboration analyses. ${ }^{11,12}$ The use of the eTICI is supported by data showing its strong correlation with clinical outcome. ${ }^{13}$ 
The mounting data from stroke trials, registries, and practice of endovascular therapy emphasize the important role of the independent assessment of a core lab for the standardized implementation of the TICI scale.
The core lab bears considerable responsibility for the impact of any endovascular stroke trial, registry or multicenter study. Device-induced therapeutic recanalization of an arterial occlusion in the cerebral circulation

TABLE 1. $\mathrm{mTICl}$ scores from the endovascular stroke trials (eTICI for ESCAPE)

\begin{tabular}{lccccc}
\hline & MR CLEAN $(n=196)$ & ESCAPE* $(n=156)$ & SWIFT PRIME $(n=83)$ & EXTEND-IA $(n=29)$ & REVASCAT $(n=102)$ \\
\hline 0 & $27(13.8)$ & $6(3.9)$ & $4(4.8)$ & $1(3)$ & $8(7.8)$ \\
1 & $11(5.6)$ & $3(1.9)$ & $1(1.2)$ & $1(3)$ & $2(2.0)$ \\
$2 a$ & $43(21.9)$ & $34(21.8)$ & $5(6.0)$ & $2(7)$ & $25(24.5)$ \\
$2 b$ & $68(34.7)$ & $75(48.1)$ & $16(19.3)$ & $11(38)$ & $48(47.1)$ \\
3 & $47(24.0)$ & $38(24.4)$ & $57(68.7)$ & $14(48)$ & $19(18.6)$ \\
$2 b$ or 3 & $115(58.7)$ & $113(72.4)$ & $73(88)$ & $25(86)$ & $67(65.7)$ \\
\hline
\end{tabular}

Values are presented as number (\%).

mTICl; modified Thrombolysis in Cerebral Infarction, eTICl; expanded Thrombolysis in Cerebral Infarction, ESCAPE; Endovascular Treatment for Small Core and Anterior Circulation Proximal Occlusion with Emphasis on Minimizing CT to Recanalization Times, MR CLEAN; Multicenter Randomized Clinical Trial of Endovascular Treatment for Acute Ischemic Stroke in the Netherlands, SWIFT PRIME; Solitaire ${ }^{\mathrm{TM}}$ With the Intention For Thrombectomy as PRIMary Endovascular Treatment, EXTEND-IA; Extending the Time for Thrombolysis in Emergency Neurological Deficits - Intra-Arterial, REVASCAT; Endovascular Revascularization With Solitaire Device Versus Best Medical Therapy in Anterior Circulation Stroke Within 8 Hours.

*The ESCAPE trial used eTICI scoring.
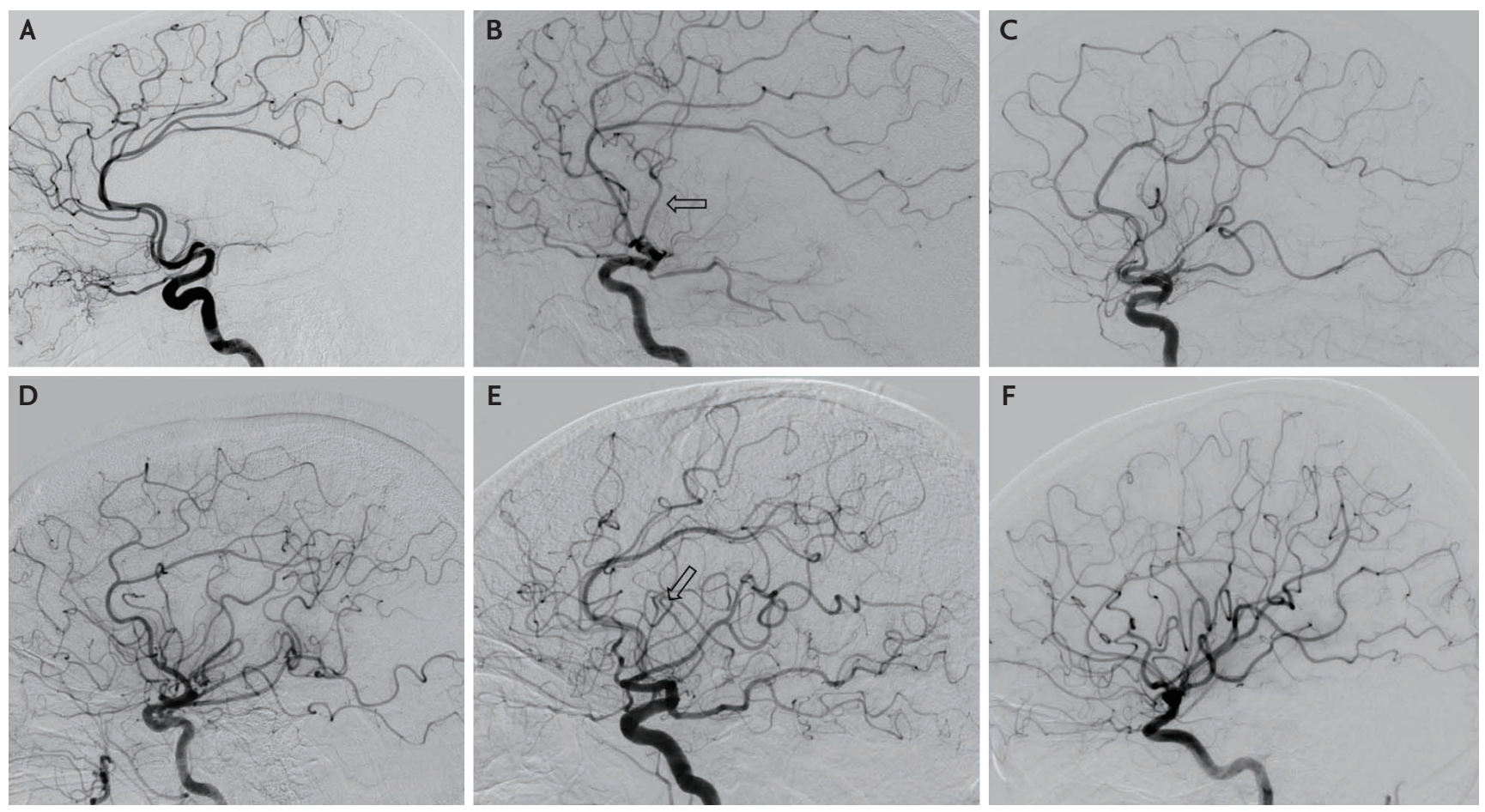

FIG. 1. Expanded treatment in cerebral ischaemia score. (A) Grade o-1 with no or minimal distal branch filling and no perfusion. (B) Grade 2a: anterograde perfusion of less than half of the middle cerebral artery (MCA) territory through small anterior MCA branch (arrow). (C) Grade $2 b$ (50): anterograde perfusion of more than half of the MCA territory but less than two thirds. (D) Grade $2 b(67)$ : anterograde perfusion of more than two thirds of the MCA territory but less than 90\%. (E) Grade 2c: near complete perfusion of the MCA territory with slow filling of one (arrow) or few distal MCA branches. (F) Grade 3: complete perfusion of the MCA territory. 
is the obvious mechanism of potential benefit, whereas the resultant reperfusion in the downstream territory is one of the key biological determinants of subsequent outcomes. All endovascular devices developed to date focus on recanalization and only indirectly culminate in reperfusion. Thus, trialists and sponsors may wish for "lenient/generous" interpretation of the TICI score by core labs as higher TICI scores (or percentages) may potentially be used for marketing or establishing the superiority of technique A vs. technique B or device A vs. device B.

A considerable gap still exists between those with angiographic success and clinical outcomes. ${ }^{14-16}$ The core lab should objectively score the angiographic results without proclivity in any regard. It should also be blinded to clinical data, divorced from any statistical analyses and properly credited for their scientific contributions. The reputation of a core lab should be founded on a track record of experience, capability in managing voluminous datasets, systematic methods free of bias, and timely interpretation of imaging. Trialists or sponsors partnership with "lenient" core labs may be counterproductive when the clinical outcomes do not mirror overrated angiographic success. Additionally, we collectively need to reduce inter-observer variability among core labs through education and accreditation. This raises the question of who is qualified to teach and/or accredit? The resulting controversy may render this approach ineffective. Another potential approach could be to create a detailed grading system that allows for many of the subtleties of image interpretation. This again may make the scale impractical since simplicity is a key determinant of the success for any scale. Finally, semi-automated quantitative methods that quantify volume and extent of collateral blush may increase robustness of perfusion assessment. This may be technically challenging given the many variables that could influence the results including rate of contrast injection, field of view, projection and length of the acquisition etc. We propose a practical solution by creating a library of cases incorporating all the common variants and to provide a consensus read by experts. This will form a 'go-to' reference library that core labs can consult for revising or comparing an individual tough case against a library of such cases. Such an expert dataset could also point to common pitfalls, errors and biases.

The efforts to enhance core labs quality can only be achieved if the exact application of the TICI scoring system are clarified. When the majority of subjects in every study achieve greater than TICI $2 \mathrm{~A}$ reperfusion, it becomes paramount that scoring systems discriminate even seemingly subtle variation in reperfusion. Such attempt was done for scoring the quality of reperfusion in the HERMES collaboration meta-analysis. The TICI-2b reperfusion was further classified into $2 \mathrm{~b}-50$, and $2 \mathrm{~b}-$ 67. ${ }^{12}$ Although the difference in outcomes between these two categories was significant, these refinements can be hard to implement in routine practice. Other Factors that can be considered include heterogeneity and topography of reperfusion, and the functional eloquence of different brain regions. On the other hand, there are potential important considerations in cases that do not achieve TICI 3 flow. Possibilities include inadequate recanalization of the original occlusion, embolus into a smaller branch distal from the original clot, or due to absence of demand from the tissue (akin to the no-reflow phenomenon: opening the vessel that is supplying dead brain). There is also the possibility that perfusion may continue to improve after the procedure aided by the restored flow and innate thrombolytic mechanisms. This may partly explain the difference in the incidence of emboli in new territory and infarcts in new territory: not every new embolus results in an infarct. ${ }^{12}$ As efforts to improve the scoring systems move forward, it is important to consider these scenarios in the development of tools to accurately assess the quality of reperfusion.

The angiography data of endovascular stroke studies have tremendous value if properly analyzed by an experienced core lab. Many lessons may be learnt on angiography, yet even the mere accurate TICI scoring carries great value. The assessment of technical strategy and efficacy of a particular device could be assessed by accurate, standardized TICI reporting. Refinements of the endovascular techniques may also be gained from detailed TICI analyses. Data sharing enables the pooling of multiple trials or studies at the individual patient level for angiographic comparisons. The stroke community should be cautious of comparisons across trials read in different core labs until identical and systematic methodology in TICI scoring is used. In routine clinical practice, the success and quality of endovascular ther- 
apy may be ill-described by local investigators scoring their own cases especially when influenced by knowledge of the clinical outcomes. Local versus central core lab readings may serve as an important educational tool and the resulting datasets may be archived as a rich resource to teach future generations. Current TICI scoring systems including eTICI do not incorporate the efficiency aspect of the procedure. As we have increasingly robust data regarding 'time is brain', ${ }^{17}$ trialists and industry may wish to incorporate a component of the ease with which a high- quality reperfusion (eTICI $2 c$ or 3) has been achieved. This could be in terms of time (e.g., within 2 minutes or reaching the carotid; 2-5 minutes or $>5$ minutes) or number of passes (one pass, two passes or more than two passes). The importance of complete reperfusion (TICI-2c or 3) with first deployment of retrievable stent, described as first pass effect, is emphasized in a recent report from the North American Solitaire Acute Stroke Registry database describing significantly higher rates of good functional outcomes with first pass effect. ${ }^{18}$ Many trials did not capture that degree of detail from the procedure, making these aspects difficult to assess. In addition, current scores do not take the primary occlusion site into consideration. To illustrate, reperfusion of an Mr-level occlusion is given equal weight to reperfusion on an M2 occlusion despite the different areas of perfusion deficit and the different outcomes of these occlusion sites.

In summary, TICI (especially the eTICI score used in the HERMES analysis) is at the core of endovascular stroke therapies, underscoring the pivotal role of a core lab. The job of a core lab is simply defined as reliably interpreting the imaging data independent of any bias, yet its impact is profound. Education especially by creating a library of cases may improve standardization. In light of the ever-increasing, large datasets, there is the potential to extract greater information from the final angiographic runs. These may be invaluable in the evaluation of new adjunctive therapies. In the meantime, we should refrain from comparing techniques, therapies, or centers based on comparing TICI scores from different core labs. Ongoing and future trials are encouraged to capture greater degree of procedural details to allow further refinement of reperfusion assessment and incorporating procedural efficiency and ease.

\section{Conflicts of Interest}

No potential conflicts of interest relevant to this article was reported.

\section{REFERENCES}

1. Berkhemer OA, Fransen PS, Beumer D, van den Berg LA, Lingsma HF, Yoo AJ, et al. A randomized trial of intraarterial treatment for acute ischemic stroke. $N$ Engl J Med. 2015;372:11-20.

2. Goyal M, Demchuk AM, Menon BK, Eesa M, Rempel JL, Thornton J, et al. Randomized assessment of rapid endovascular treatment of ischemic stroke. N Engl J Med. 2015;372:1019-1030.

3. Saver JL, Goyal M, Bonafe A, Diener HC, Levy EI, Pereira $\mathrm{VM}$, et al. Stent-retriever thrombectomy after intravenous t-PA vs. t-PA alone in stroke. N Engl J Med. 2015;372:22852295.

4. Campbell BC, Mitchell PJ, Kleinig TJ, Dewey HM, Churilov L, Yassi N, et al. Endovascular therapy for ischemic stroke with perfusion-imaging selection. N Engl J Med. 2015;372:1009-1018.

5. Jovin TG, Chamorro A, Cobo E, de Miquel MA, Molina CA, Rovira A, et al. Thrombectomy within 8 hours after symptom onset in ischemic stroke. N Engl J Med. 2015;372:22962306.

6. Zaidat OO, Yoo AJ, Khatri P, Tomsick TA, von Kummer R, Saver JL, et al. Recommendations on angiographic revascularization grading standards for acute ischemic stroke: a consensus statement. Stroke. 2013;44:2650-2663.

7. Higashida RT, Furlan AJ, Roberts H, Tomsick T, Connors B, Barr J, et al. Trial design and reporting standards for intra-arterial cerebral thrombolysis for acute ischemic stroke. Stroke. 2003;34:e109-e137.

8. Saver JL, Liebeskind DS, Nogueira RG, Jahan R. Need to clarify thrombolysis in myocardial ischemia (TIMI) scale scoring method in the penumbra pivotal stroke trial. Stroke. 2010;41:e115-e116.

9. Yoo AJ, Simonsen CZ, Prabhakaran S, Chaudhry ZA, Issa MA, Fugate JE, et al. Refining angiographic biomarkers of revascularization: improving outcome prediction after intra-arterial therapy. Stroke. 2013;44:2509-2512.

10. Shuaib A, Butcher K, Mohammad AA, Saqqur M, Liebeskind DS. Collateral blood vessels in acute ischaemic stroke: a potential therapeutic target. Lancet Neurol. 2011;10:909-921. 
11. Goyal M, Fargen KM, Turk AS, Mocco J, Liebeskind DS, Frei $\mathrm{D}$, et al. $2 \mathrm{C}$ or not $2 \mathrm{C}$ : defining an improved revascularization grading scale and the need for standardization of angiography outcomes in stroke trials. J Neurointerv Surg. 2014;6:83-86.

12. Liebeskind DS, Bracard S, Guillemin F, Jahan R, Jovin TG, Majoie CB, et al. eTICI reperfusion: defining success in endovascular stroke therapy. J Neurointerv Surg. 2018 Nov 24. [E-pub]. http://dx.doi.org/10.1136/neurintsurg-2018-014110.

13. Almekhlafi MA, Mishra S, Desai JA, Nambiar V, Volny O, Goel A, et al. Not all "successful" angiographic reperfusion patients are an equal validation of a modified TICI scoring system. Interv Neuroradiol. 2014;20:21-27.

14. IMS II Trial Investigators. The interventional management of stroke (IMS) II study. Stroke. 2007;38:2127-2135.

15. Del Zoppo GJ, Higashida RT, Furlan AJ, Pessin MS, Rowley HA, Gent M. PROACT: a phase II randomized trial of recombinant pro-urokinase by direct arterial delivery in acute middle cerebral artery stroke. PROACT Investigators. Prolyse in acute cerebral thromboembolism. Stroke. 1998;29:4-11.

16. Hussein HM, Georgiadis AL, Vazquez G, Miley JT, Memon MZ, Mohammad YM, et al. Occurrence and predictors of futile recanalization following endovascular treatment among patients with acute ischemic stroke: a multicenter study. AJNR Am J Neuroradiol. 2010;31:454-458.

17. Saver JL, Goyal M, van der Lugt A, Menon BK, Majoie CB, Dippel DW, et al. Time to treatment with endovascular thrombectomy and outcomes from ischemic stroke: a meta-analysis. JAMA. 2016;316:1279-1288.

18. Zaidat OO, Castonguay AC, Linfante I, Gupta R, Martin CO, Holloway WE, et al. First pass effect: a new measure for stroke thrombectomy devices. Stroke. 2018;49:660-666. 